\title{
Mycobacterium tuberculosis Beijing outbreak in a school in Marseille, France, 2012
}

F Golesi ${ }^{1}$, J Brignatz ${ }^{2}$, M Bellenfant ${ }^{2}$, D Raoult ${ }^{1}$, M Drancourt (michel.drancourt@univmed.fr) ${ }^{1}$

1. Aix Marseille Université, URMITE, UMR63, CNRS 7278, IRD 198, INSERM 1095, 13005 Marseille, France

2. Centre de Lutte Anti-Tuberculeuse du Conseil Général 13, Dispensaire d’Arenc-Bougainville, Marseille, France

Golesi F, Brignatz J, Bellenfant M, Raoult D, Drancourt M. Mycobacterium tuberculosis Beijing outbreak in a school in Marseille, France, 2012. Euro Surveill. 2013;18(2):pii=20354. Available online: http://www.eurosurveillance.org/ViewArticle.aspx?Articleld =20354

Between January and September 2012, a teacher and four students at a technical college in Marseille, France, developed pulmonary tuberculosis. All Mycobacterium tuberculosis isolates from these cases were identical and belonged to the Beijing strain family, multispacer sequence type 72 , a rare genotype identified only once in our laboratory in the previous two years. This report highlights once more the potential for $M$. tuberculosis Beijing strains to cause outbreaks, this time in a school setting.

Among the different genotypes of Mycobacterium tuberculosis pathogenic to humans, the Beijing strains - first described 20 years ago in the Chinese capital are now known to have a global distribution $[1,2]$. The Beijing strain family is characterised by its potential to cause epidemics and its association with drug resistance $[3,4]$. Several outbreaks of pulmonary tuberculosis (TB) due to $M$. tuberculosis Beijing have been reported in congregate settings such as homeless shelters [5] and prisons [6]. We report on a recent outbreak of pulmonary TB cases attributed to M. tuberculosis Beijing genotype in a school.

\section{Outbreak description}

In January 2012, a 20-year-old male (Case 1) attending a technical school in Marseille, in southern France, was diagnosed with pulmonary TB and mediastinal lymph node involvement. Direct microscopy examination of three sputum specimens did not detect acid fast bacilli (AFB) but M. tuberculosis was cultured from the sputa and broncho-alveolar fluid. The strains were susceptible to rifampicin and isoniazid and the patient was successfully treated using a combination of rifampicin, isoniazid, pyrazinamide and ethambutol. In mid-June 2012, a 42-year-old teacher at the same school (Case 2) was also diagnosed with pulmonary TB. He presented with fever and weight loss as well as a cough that had lasted for the previous six months. Radiology showed extensive lesions in both lungs with multiple cavitations. Three sputum samples yielded an average of 100 AFB per microscopy field and $M$. tuberculosis susceptible to first-line anti-tuberculosis drugs was cultured.
Response to first-line drug combination therapy was favourable and no AFB were detectable on sputum microscopy after two months. In early July 2012, as part of the investigation of these two cases by the local public health authorities for TB control, pulmonary TB was diagnosed in a second student in the same school (Case 3) who presented both clinical and radiological signs. The diagnosis was confirmed by culture from sputum specimens, which also isolated drug-susceptible M. tuberculosis.

\section{Contact tracing}

These cases of pulmonary TB clustered in time and place led to a broader investigation including a total of 334 individuals associated with this school in 2012, comprising students, teachers and administrative personnel. All were tested with interferon gamma release assays (IGRA) and 30 tests were interpreted as positive. Those who tested positive were put on prophylactic medication with isoniazid. Family members of the three patients who had been in regular contact with the three and 20 other persons in a factory where Case 1 was a trainer were also examined by symptom screening, followed by X-ray, and then sputum microscopy. In September 2012, this investigation led to the detection of subclinical pulmonary TB in two students (Cases 4 and 5) attending the same class as Case 3. In both cases, culture of respiratory tract samples yielded drug-susceptible $M$. tuberculosis isolates.

The investigation led to the hypothesis that the teacher (Case 2) was the source case of this outbreak. The source case had no identified risk for TB from significant contact outside the school or a previous history of TB. Given that his clinical signs and symptoms were initially attributed to chronic obstructive pulmonary disease secondary to heavy smoking, the diagnosis of TB was delayed and only confirmed after that of Case 1. Cases 3 and 4 were considered to have been exposed in the class in which the teacher taught. In addition, the teacher gave truck-driving lessons to the students of this class three times per week with each session lasting four consecutive hours. No direct contact between Case 1 and the teacher was established. 


\section{Laboratory investigations}

M. tuberculosis isolates collected in all five cases were sent to our reference laboratory for further analyses. Molecular testing using a marketed assay (GenExpert, Cepheid, Maureus-Scoport, France) [7] confirmed the identification of $M$. tuberculosis and detected no mutations associated with rifampicin resistance. Susceptibility testing using conventional liquid culture showed no resistance to all first-line antimycobacterials. Real-time PCR genotyping targeting the intergenic region Rvo927c-pstS3 [8] and a second region specific to the Beijing family [9] confirmed a Beijing genotype for all isolates. Multispacer sequence-typing (MST) [10] identified a genotype MST72 and confirmed that the isolates were indistinguishable. Our laboratory had only detected this genotype once previously among 174 strains of $M$. tuberculosis genotyped since 2010 (data not shown) in a patient born in Thailand and residing in France who had no known contacts with the cases reported in the current outbreak.

\section{Conclusions}

To our knowledge, this is the first report of an outbreak of $M$. tuberculosis Beijing genotype infections occurring in a school. This is also the largest outbreak of pulmonary TB detected in Marseille area in the last 15 years: in this area, the other outbreaks have only occurred in penitentiary establishments. This report illustrates the highly pathogenic and highly contagious nature of the Beijing strain family, which requires a quick laboratory confirmation. This outbreak was characterised by the high contagiousness of the source case and a clinically-severe picture in three of the five cases detected. Prolonged contact within the confines of a truck cabin (with an approximate volume of six cubic metres) may confer a risk of TB infection not very different from that in a long-haul airplane flight [11]. This outbreak also illustrates the importance of contact tracing and laboratory investigation in improving the early detection of linked cases of TB and the need to maintain a high index of suspicion for this disease even in the presence of other conditions leading to chronic cough. As in this report, most cases of TB today are caused by organisms which are not drug-resistant and which can be cured using a standard treatment with combination of anti-TB drugs for at least six months [12]. Patients on treatment only require isolation until they are no longer infectious. Given that Beijing strains are particularly transmissible and often exhibit multi-drug resistance, France and other European countries are concerned with Beijing strain family of $M$. tuberculosis [13]. Schools should be integrated in nation-wide surveillance systems for TB in France.
References

1. Van Soolingen D, Qian L, de Haas PE, Douglas JT, Traore $\mathrm{H}$, Portaels $\mathrm{F}$, et al. Predominance of a single genotype of Mycobacterium tuberculosis in countries of East Asia. J Clin Microbiol. 1995;33(12):3234-8.

2. Bifani PJ, Mathema B, Kurepina NE, Kreiswirth BN. Global dissemination of the Mycobacterium tuberculosis W-Beijing family strains. Trends Microbiol. 2002;10(1):45-52.

3. Glynn JR, Whiteley J, Bifani PJ, Kremer K, van Soolingen D. Worldwide occurrence of Beijing/W strains of Mycobacterium tuberculosis: a systematic review. Emerg Infect Dis. 2002;8(8):843-9.

4. Hanekom M, Gey van Pittius NC, McEvoy C, Victor TC, Van Helden PD, Warren RM. Mycobacterium tuberculosis Beijing genotype: a template for success. Tuberculosis (Edinb). 2011;91(6):510-23.

5. Munsiff SS, Nivin B, Sacajiu G, Mathema B, Bifani P Kreiswirth BN. Persistence of a highly resistant strain of tuberculosis in New York City during 1990-1999. J Infect Dis. 2003;188(3):356-63.

6. Ignatova A, Dubiley S, Stepanshina V, Shemyakin I. Predominance of multi-drug-resistant LAM and Beijing family strains among Mycobacterium tuberculosis isolates recovered from prison inmates in Tula Region, Russia. J Med Microbiol. 2006;55(Pt10):1413-8.

7. Dorman SE, Chihota VN, Lewis JJ, Shah M, Clark D, Grant $A D$, et al. Performance characteristics of the Cepheid Xpert MTB/RIF test in a tuberculosis prevalence survey. PLoS One. 2012;7(8):e43307.

8. Leung ET, Zheng L, Wong RY, Chan EW, Au TK, Chan RC, et al. Rapid and simultaneous detection of Mycobacterium tuberculosis complex and Beijing/W genotype in sputum by an optimized DNA extraction protocol and a novel multiplex realtime PCR. J Clin Microbiol. 2011;49(7):2509-15.

9. Hillemann D, Warren R, Kubica T, Rüsch-Gerdes S, Niemann S. Rapid detection of Mycobacterium tuberculosis Beijing genotype strains by real-time PCR. J Clin Microbiol. 2006;44(2):302-6.

10. Djelouadji Z, Arnold C, Gharbia S, Raoult D, Drancourt M. Multispacer sequence typing for Mycobacterium tuberculosis genotyping. PLos One. 2008;3(6):e2433.

11. Kenyon TA, Valway SE, Ihle WW, Onorato IM, Castro KG. Transmission of multidrug-resistant Mycobacterium tuberculosis during a long airplane flight. N Engl J Med. 1996;334(15):933-8.

12. World Health Organization (WHO). Treatment of Tuberculosis: guidelines for national programmes. 4th edition. Geneva: WHO. 2009. Available from: http://whqlibdoc.who.int/ publications/2010/9789241547833_eng.pdf

13. Cohen-Bacrie S, Ben Kahla I, Botelho-Nevers E, Million $M$, Parola $P$, Brouqui $P$, et al. Imported extensively drug. resistant Mycobacterium tuberculosis Beijing genotype, Marseilles, France, 2011. Euro Surveill. 2011;16(16):pii=19846. Available from: http://www.eurosurveillance.org/ViewArticle. aspx?Articleld $=19846$ 